\title{
UNUSUAL PRESENTATIONS IN INGUINAL HERNIA: FOUR CASES WITH REVIEW OF LITERATURE.
}

R. M. Prajapati, S. M. Datey, Rishikant Vashishtha, Anurag Shrivastav, Vikramaditya Singh

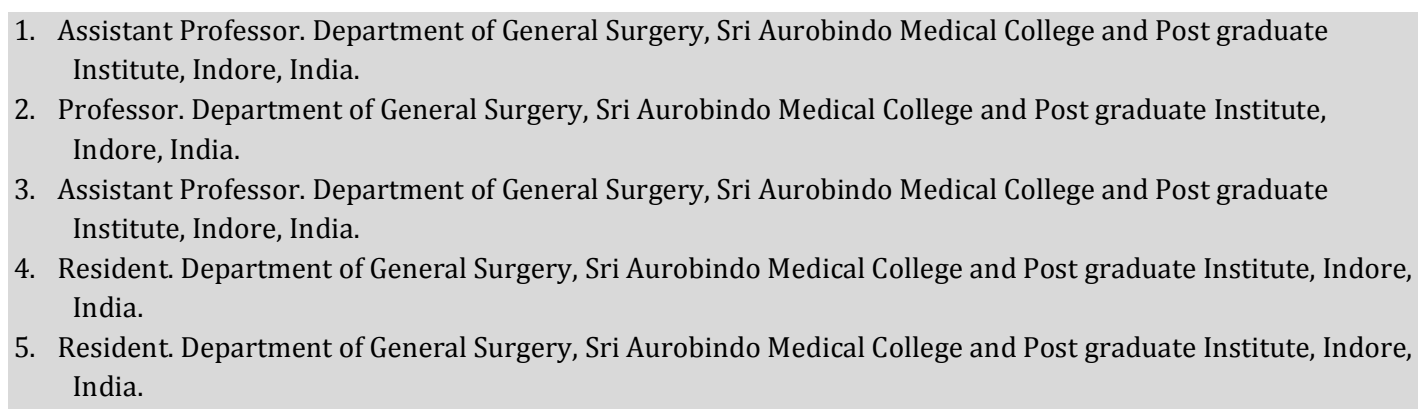

\section{CORRESPONDING AUTHOR:}

Dr. R. M. Prajapati, M. S.

260, Vidyapati Nagar,

Nanakheda, Ujjain (M.P.)

E-mail: gunjan8prajapati@gmail.com

ABSTRACT: Inguinal Hernia is a very common clinical entity with varied presentations. We are presenting four unusual cases of inguinal hernias, who came to us at The Sri Aurobindo Medical College and Postgraduate Institute at Indore. Out of the four cases three presented with incarcerated inguinal hernias in emergency, while one was a routine case. They presented with following unusual features; (1)Strangulated Meckel's Diverticulum as content (2) Inflamed Appendix Vermicularis as content (3) Strangulated omentum showed metastasis of a hidden intraabdominal adenocarcinoma (4) Beaded hernial sac which revealed mesothelial hyperplasia on histopathology.

Abdominal hernia is the result of abnormal protrusion of abdominal content through a defect in the abdominal wall. Its treatment is the surgical repair of the defect with satisfactory results. Although these unusual presentations of contents have no implications on the outcome of its surgical repair, but one should be aware of such possibility at exploration. We have presented these cases with review of literature as it will be beneficial to all .

CASE 1: Nineteen year old male presented with incarcerated right inguinal hernia, which on exploration was found have a Strangulated Meckel's Diverticulum. Resection of ileal segment bearing the Meckel's Diverticulum, with an end to end ileal anastomosis done. Posterior wall of the inguinal canal was repaired by herniorrhaphy. Postoperative recovery was uneventful. It was a case of Strangulated Littre's Hernia.

CASE 2: Eighteen year old male presented with right inguinal hernia with pain. There was no history of vomiting or fever.Hematological investigations showed polymorphonuclear leucocytosis. On exploration the sac had the Appendix Vermiformis as its content. Appendectomy and repair of hernia by herniorrhaphy was done. Appendix showed congestion and mesentery was oedematous. Postoperative recovery was uneventful and patient was discharged on 7 th postoperative day. Histopathological examination revealed 
features of acute appendicitis in the specimen of the appendix. This was a case of Amayand's Hernia.

CASE 3: Sixty five year old male was admitted with Left incarcerated inguinal hernia with pain and difficulty in walking The patient did not have any other complaint. Physical examination and routine investigations were within normal limits. On exploration, sac of the hernia had strangulated omentum. Hernial defect was repaired by hernioplasty with prolene mesh. Excised omentum and sac were sent for the routine histopathological examination which showed deposits of metastasis from hidden intraabdominal adenocarcinoma.This was a case of metastatic adenocarcinoma in an incarcerated omentum in inguinal hernia.

CASE 4: Twenty nine year old male was operated for right inguinal hernia. Hernial sac was atypical. Peritoneum forming the sac was beaded and rough. Although there were no adhesions with the omentum but it was thickened. Hernioplasty with prolene mesh was done after excising the sac. Histopathological examination revealed features of mesothelial hyperplasia in the excised sac of this hernia.

DISCUSSION AND REVIEW OF LITERATURE: Hernia is the result of a defect in the abdominal wall. Surgical treatment is complete when the defect is closed efficiently. Contents of an abdominal hernia usually are small bowel, omentum, ovary and fallopian tube Unusually any peritoneal organ can present as a content of a hernial sac. Review of literature shows many unusual presentations with varied contents.

In our series of such unusual presentations the first case is that of Littre's hernia i. e. a hernial sac containing a Meckel's Diverticulum. Littre's hernia is defined as the protrusion of the Meckel's Diverticulum through a potential abdominal opening(Ref 1 ). Littre's Hernia is uncommon and a detailed review of literature revealed that very few cases of a strangulated Littre's hernia are reported (ref 2 ).Reviewed literature and this case report shows that Meckel's Diverticulum can be safely resected with repair of hernia. Multiple pathologies encountered in the course of an abdominal operation should be dealt with, on its own merits and established principles of surgery.

Amayand's hernia is named after Claudius Amayand (1680-1740), an English surgeon who did the first recorded successful appendectomy(Ref3). When a hernial sac contains vermiform appendix, inflamed or un-inflammed, it is called as an Amayand's Hernia. True incidence of this condition is difficult to estimate as all the cases are not registered. Ryan WJ et al(Ref 4) have reported only 11 cases of appendicitis out of 8692 $(0.13 \%)$ cases of hernial sacs with appendix. D. Alia et al observed presence of appendicitis in $0.8 \%$ of 1341 operations of inguinal hernia. Vermiform appendix can be safely removed and hernia can be repaired in the usual way.

Case (3) showed presence of metastatic deposits of an adenocarcinoma in the excised omentum from the hernial sac. (Ref 5) Mayo clinic's retrospective study on inguinal hernias (1950-1988) reported that only 15 out of 22816 patients had metastatic deposits in their hernial sacs (0.05\%). An intraabdominal malignancy can spread to omentum or peritoneum of the hernial sac by direct contiguity or by transcoelomic implantation. The hernial sac can be involved in three ways namely intrasaccular, saccular and extrasaccular (Ref 6) Extrasaccular involvement of sac is by the malignancy of a nearby structure for example a malignant inguinal lymph node infiltrating a sac of an inguinal hernia. Saccular is the primary mesothelioma of peritoneum. Omentum with 
secondary deposits is intrasacular involvement as it was in the reported case. Other examples of intrasacular involvement are malignancies of appendix, colon, and ovary when these organs with malignancy become a content of the hernial sac(Ref 7). This case emphasizes the importance of histopathological examination of hernial sacs and contents removed at operation(ref 8 )

Case (4) is that of nodular mesothelial hyperplasia in a hernial sac. Mesothelial hyperplasia results in formation of nodules over the wall of the sac. Nodules can be lying free or can be attached to the wall of sac. Histopathologically these nodules are formed exclusively of one cell type. These cells show moderate degree of anisocytosis and pleomorphism. Rosai J et al (ref 9) reported a series of thirteen cases of hernial sac exhibiting mesothelial hyperplasia of such a degree as to simulate a malignancy i.e. mesothelioma. Mesothelioma has to be differentiated with mesothelial hyperplasia by immunohistochemistry.

Review of literature regarding unusual presentations in inguinal hernias gave us an insight of many clinically interesting cases. Intraperitoneal or retroperitoneal haemorrhages as well as many other congenital, infectious, or neoplastic processes occuring in the vicinity of groin may present within an inguinal hernia.

Gibbon CE et al(ref 10) reported a rare presentation where a dilated stomach of gastric outlet obstruction became a content of inguinal hernia. Sherman HF et al (ref 11) reported a patient presenting with incarcerated inguinal hernia which turned out to be collected blood due to a bygone splenic rupture. Mayank Jain et al(ref 12) had an inflamed appendage epiploicae in an incarcerated left inguinal hernia on trans peritoneal pre peritoneal (TAPP) hernioplasty.

Nishikant $N$ Gujar et al (ref 13) reported a case of male form of persistent Mullerian Duct Syndrome of type I (hernia uteri inguinalis) which presented as an obstructed inguinal hernia. Prasannan S et al(ref 14) have documented one case with a ruptured ectopic pregnancy presenting as an irreducible inguinal hernia. Seema Khanna et al(ref15) described a case where an atrophied uterus was the content of the sac of an inguinal hernia in an adult female.

Aberrant Adrenocortical tissue was found in the hernial sac of a male by Demellawy et al(ref 16). Smithi Sripathi et al (ref 17) reported a case of left inguinal hernia in a new born with left ureter as its content. Diagnosis was done by an intravenous urography, as interesting earlier ultrasonography could not detect. A dress maker's pin was swallowed accidentally by a 18 month old boy. this pin was removed from inguinal hernial sac after two weeks(ref 18).

We conclude with quote by Sherman HF et al "Swelling in groin may represent much more than an inguinal hernia and an inguinal hernia may be much more complicated than it seems upon superficial consideration. Intraperitoneal or retroperitoneal hemorrhages, as well as many other congenital, inflammatory, infectious, or neoplastic processes occurring either locally or at distance from the groin may present in the groin, simulating a hernia or within an inguinal hernia itself" (ref 19). This list of unusual findings is endless and many more will be added in the future.

ACKOWLEDGMENT: We are grateful to Dr.Vinod Bhandari, Chairman, Sri Aurobindo Medical College and postgraduate Institute, Our Dean Dr.S.D.Joshi and Head of the Department of Surgery Dr.P.K.Banerjee for the permission to publish this article. 


\section{REFERENCES:}

1. Skandalakis PN, Oras O,Skandalakis JE, Mirilas P. Littre Hernia: Surgical Anatomy, Embryology, Technique of repair. Am Surg 2006 Mar ;72(3): 238-43

2. SM Datey, Ajay Lunawat, Avinash Vishwani, Rishikant Vashistha,Akshay Sharma . Strangulated Littre's Hernia :A case report and review of literature . Journal of evolution of medical and dental sciences. 2013 March 26-31(10)

3. D'Alia C, lo Schiavo MG, Tonante A, Taranto F, Gaglino E,Bonnano L, Di Giuseppe G,Pagano D,Stuniolo G. Amayand's Hernia:A case report and review of literature. Hernia 2003;(7) : 89-91

4. Ryan WJ. Hernia of Vermiform Appendix . Ann Surg 1937 ;106:135-139

5. Nicolson CP, Donohue JH, Thompson GB, Lewis JE. A study of metastatic cancer found during inguinal hernia repair. Cancer 1992; 69: 3008-11

6. Lejar J. Neoplasmes herniaires et peri herniaires. Gaz de hop Par . 1889 ;62 : 801-811

7. Patel T, Viswanathan S, Jambhekar NA . Metastatic adenocarcinoma presenting as an inguinal hernia: A case report with review of literature. Indian J Pathol Microbiol. 2007 Jul ; 50 (3) :541-2

8. RM Prajapati,SM Datey, Rakesh Shishodiya, Vikramaditya Singh, Himanshu Bansal . Metastatic adenocarcinoma in omentum in an incarcerated inguinal hernia: A case report with review of literature. Journal of evolution of medical and dental sciences 2013March. 05-11(26)

9. Rosai J, Dehner LP. Nodular mesothelial hyperplasia in hernia sac: a benign reactive condition simulating a neoplastic process. Cancer. 1975 Jan; 35(1);165-75

10. Gibbons CE, Malhotra AK, Harvey MH. Inguinal hernia: An unusual cause of gastric outlet obstruction. Br J Hosp Med. 1994; $52: 360-1$

11. Sherman HF. The inguinal hernia: not always straight forward, not always a hernia. J Emerg Med 1989 Jan-Feb; 7(1) :21-4

12. Mayank Jain, Shashi Khanna, Bimlendu Sen, and Om Tantia. Irreducible inguinal hernia with appendices epiploicae in the sac. J Minim Access Surg. 2008 Jul-Sep ;4(3):85-87

13. Nishikant N Gujar, Ravikumar K Choudhary, Geeta R Choudhary, Nasheen N Bagli, Harish S Mane, Jilani S Awati and Vipin Balchandran. Male form of persistent Mullerian duct syndrome type I(hernia uteri inguinalis) presenting as an obstructed inguinal hernia:a case report. 2011.5:586 doi 10.1186/1752-1947-5586

14. Prasannan s, Jabar MF, Gul YA. Ruptured Ectopic Pregnancy presenting as an irreducible inguinal hernia. Acta Chir Belg. 2004 Oct;104(5):591-2

15. Seema Khanna, Shashi Prakash Mishra, Seema Singh and Sanjeev Kumar Gupta. rare presentation of inguinal hernia in female;A case report. world Journal of Surgical Research 2013;2:2

16. D El Demellawy,a Nasr, A.Samkari,P Pastolero, S Alowami. aberrant adrenocortical tissue in hernial sac occuring in an adult: Case report and review of literature. Hernia 2009 dec;13(6):659-662

17. Smithi Sripathi, KV Rajagopal, Chandan Kakkar and Ashwin Polnaya:Case reportInguinoscrotal ureteral hernia diagnosed on micturating cystourethrography .Indian J Radiol Imaging. 2011 Jul-Sep ; 21(3); 199-121

18. Miyano Go, Takahashi, Toshiaki, Takahashi, Tsubasa, Lane, Geoffrey J, Kato, Yoshifumi, Okazaki, Tadaharu, Yamataka, Atsuyki. An unusual foreign body in an inguinal hernia sac ; case report and literature review. African journal of Paediatric Surgery. 2012 
Jan-April ; 9 (1):p 66

19. K Ballas, Th Kontoulis, Ch Skouras, A Triantafyllou, N Symeonidis, Th Pavlidis, G Marakis and A Sakadamis:unusual findings in inguinal hernia surgery:Report of 6 rare cases. Hippokratia 2009 Jul-Sep; 13(3):169-171 PMCID : PMC2765295

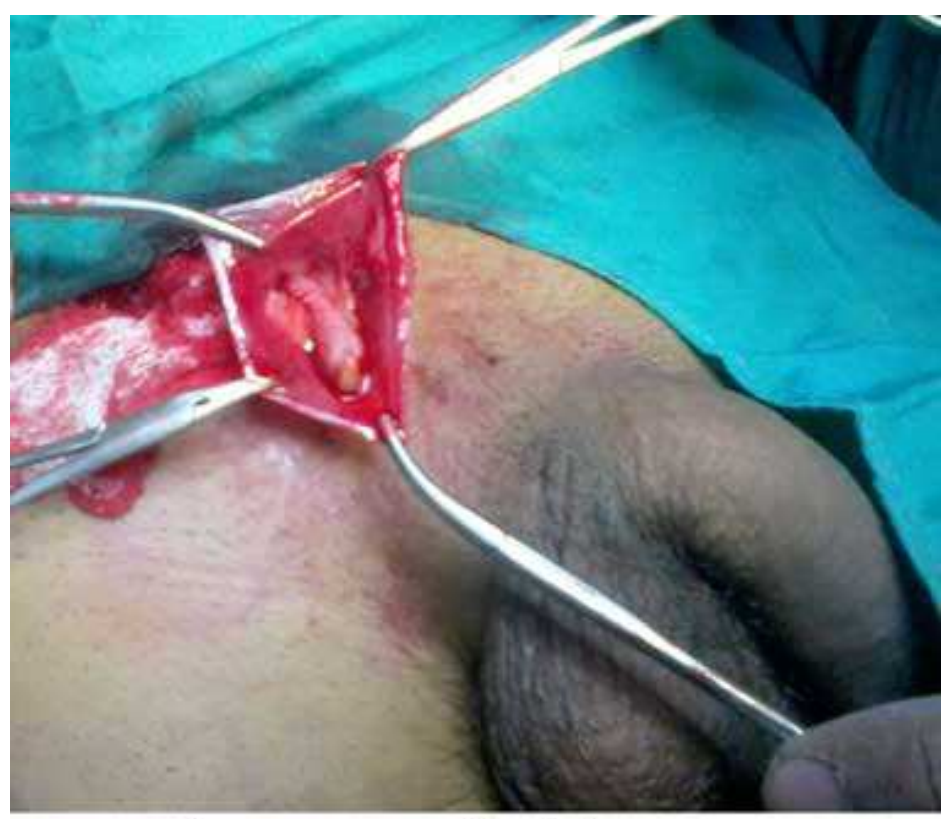

\section{Amayand's Hernia}

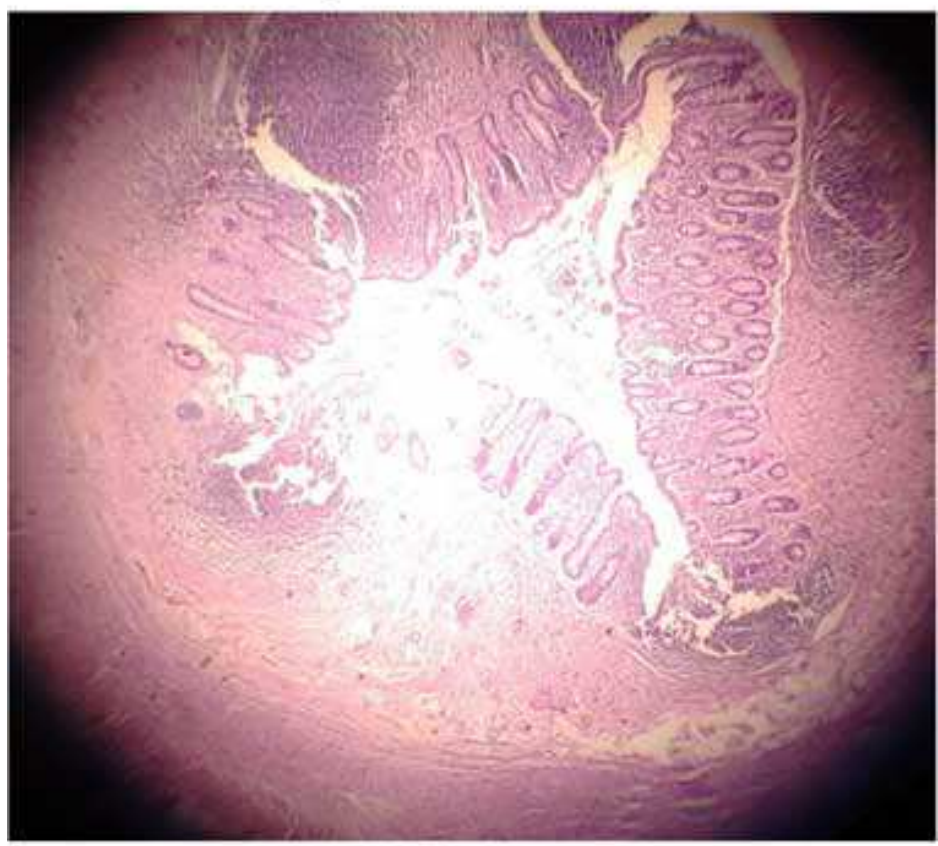

\section{Microscopic View of Appendix}




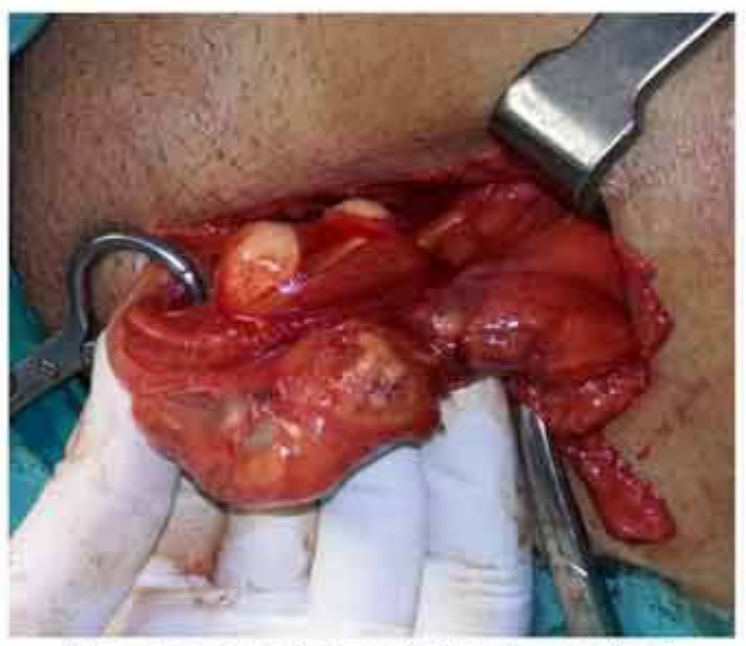

Intra operative photgraph showing involved omentum in left inguinal hernia

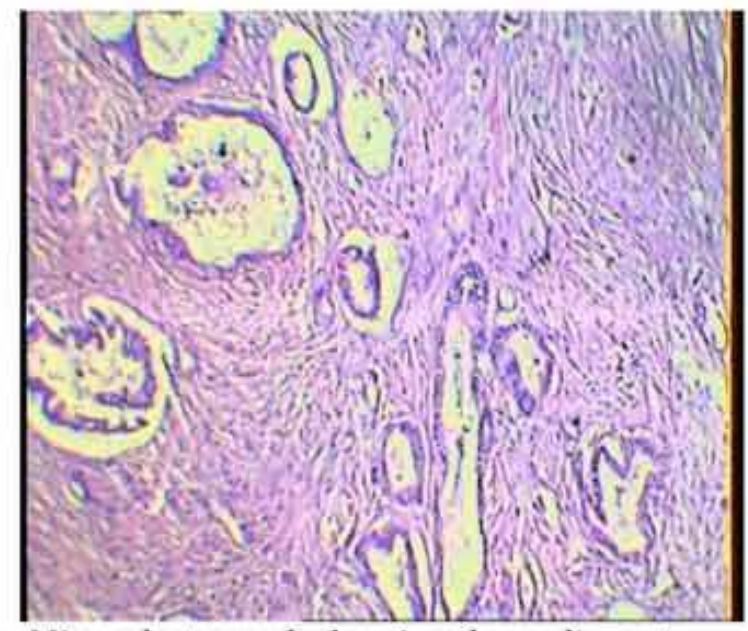

Microphotograph showing the malignant acinar structures infiltrating the fatty tissue of omentum $100 x$ Hematoxilin and eosin stains.

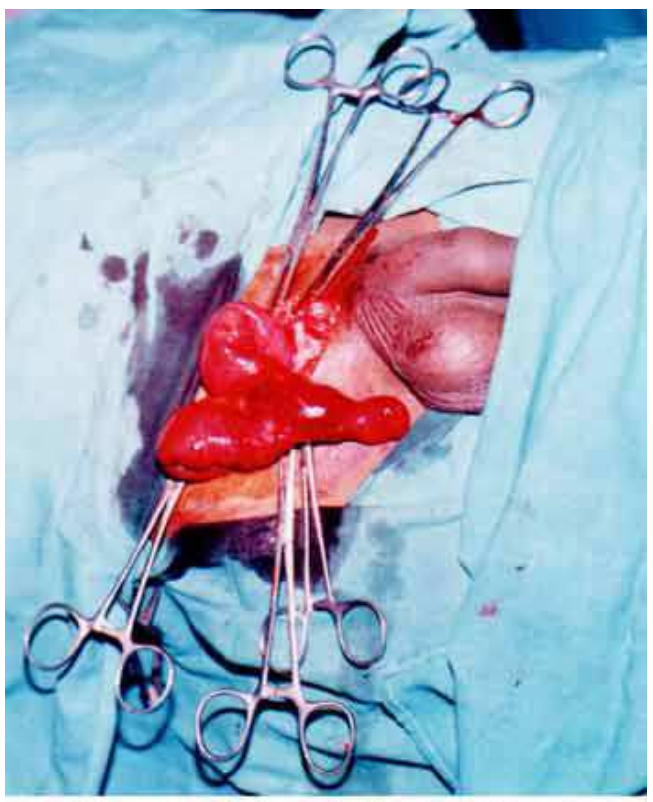

Strangulated Littre's Hernia 


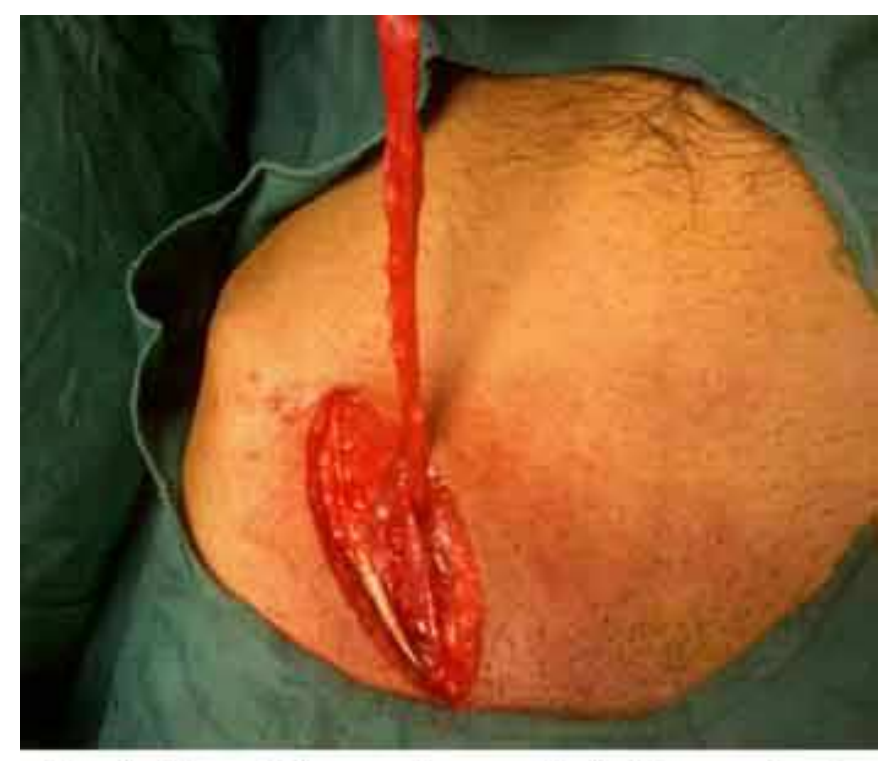

\section{Beaded hernial sac of mesothelial hyperplasia}

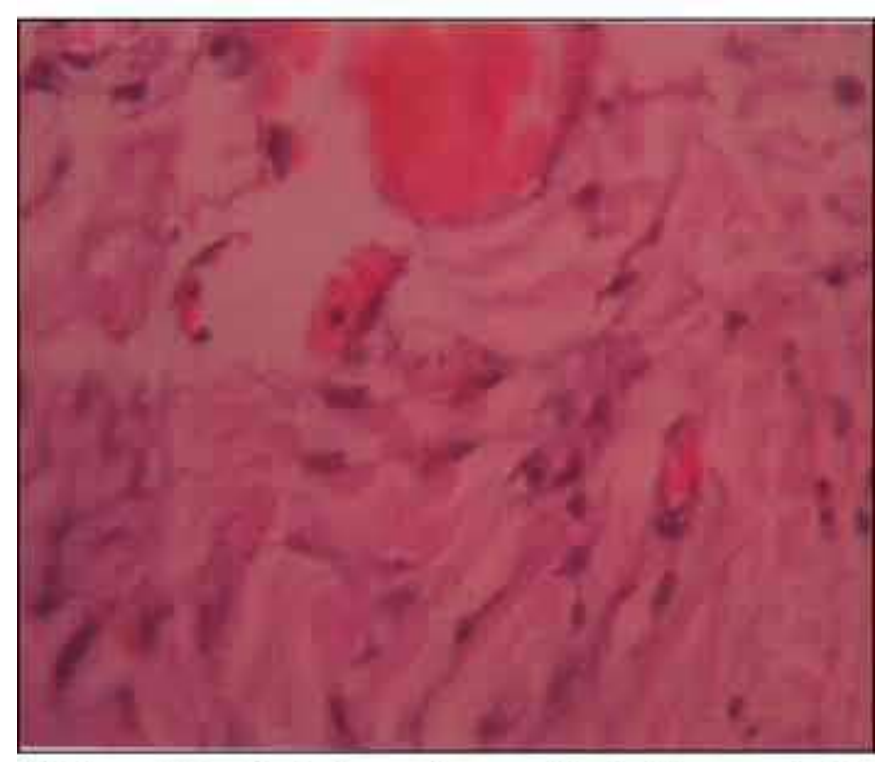

Histopathlogical view of mesothelial hyperplasia 